\title{
Free-flap Treatment of Congenital Pressure Necrosis of the Forearm in the Neonate
}

\author{
Ji Son, $\mathrm{MD}^{1,2}$ Daniel A. Medalie, $\mathrm{MD}^{1,2}$ \\ ${ }^{1}$ Division of Plastic and Reconstructive Surgery, The MetroHealth \\ System, Cleveland, Ohio \\ 2 Department of Plastic and Reconstructive Surgery, University \\ Hospitals Case Medical Center, Cleveland, Ohio
}

\begin{abstract}
Address for correspondence Ji Son, MD, Department of Plastic and Reconstructive Surgery, University Hospitals Case Medical Center, 11100 Euclid Avenue, Cleveland, OH 44106 (e-mail: ji.son@uhhospitals.org).
\end{abstract}

J Reconstr Microsurg Open 2016;1:29-31.

\begin{abstract}
Keywords

- microsurgery

- neonatal microvascular reconstruction

- congenital pressure necrosis

Background Microvascular surgery is not commonly performed in pediatric or neonatal populations, and when it is performed, it is done with some trepidation due to small size of the vessels, prolonged anesthesia, vasospasm, and difficult postoperative care. We describe microsurgical reconstruction of full-thickness loss of the forearm tissue in a 2-week-old due to congenital necrosis.

Methods A male infant was born with blistering of the ulnar aspect of the forearm. Removal of necrotic tissue resulted in a defect of $60 \%$ of his forearm skin and musculature with exposed ulna, ulnar nerve, and artery. Soft tissue defect was reconstructed using rectus free flap and split-thickness skin graft from the buttock.

Results The flap and graft survived without complication and the patient demonstrated a completely healed wound.

Conclusion With advances in operative microscopes, instruments, surgical techniques, and anesthetic techniques, free tissue transfer in the neonate is feasible despite small vascular caliber. An experienced surgical team remains one of the most important factors to obtain a good outcome.
\end{abstract}

Pediatric microsurgery was once considered impossible because of technical difficulties inherent in the small size of the vessels, prolonged anesthesia, vasospasm, and difficult postoperative care. However, with advances in operative microscopes, instruments, surgical techniques and anesthetic techniques, these problems could be largely overcome. Ohmori et al described a groin free flap on a 3-month-old. ${ }^{1}$ Parry et al outlined microsurgical tissue transfer in pediatric patients, with the youngest patient being 2 years old. ${ }^{2}$ This report describes microsurgical treatment of a full-thickness loss of the forearm tissue in a 2-week-old due to congenital necrosis. This is the youngest patient in the literature to have received successful free tissue transfer.

received

January 10, 2016

accepted after revision

April 3, 2016

published online

May 23, 2016 10.1055/s-0036-1584223. ISSN 2377-0813.

\section{Materials and Methods}

Congenital necrosis of the upper extremity is a rare and deforming phenomenon. The etiology can be diverse and frequently indeterminate, but the end result is a neonate with a soft tissue defect of the hand and/or forearm.

A male infant weighing $6 \mathrm{lbs} .7 \mathrm{oz}$. was born with blistering of the ulnar aspect of the forearm. Within several days, it became clear that the skin over the ulnar forearm was nonviable, and the child was taken to the operating room (OR) for debridement. During debridement, it was found that the underlying forearm musculature was also nonviable. It is unclear why the tissue was necrotic but a predelivery ultrasound showed the neonate's arm wedged against its head. Of

Copyright $\odot 2016$ by Thieme Medical Publishers, Inc., 333 Seventh Avenue, New York, NY 10001, USA.

License terms

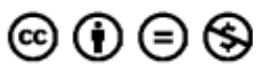




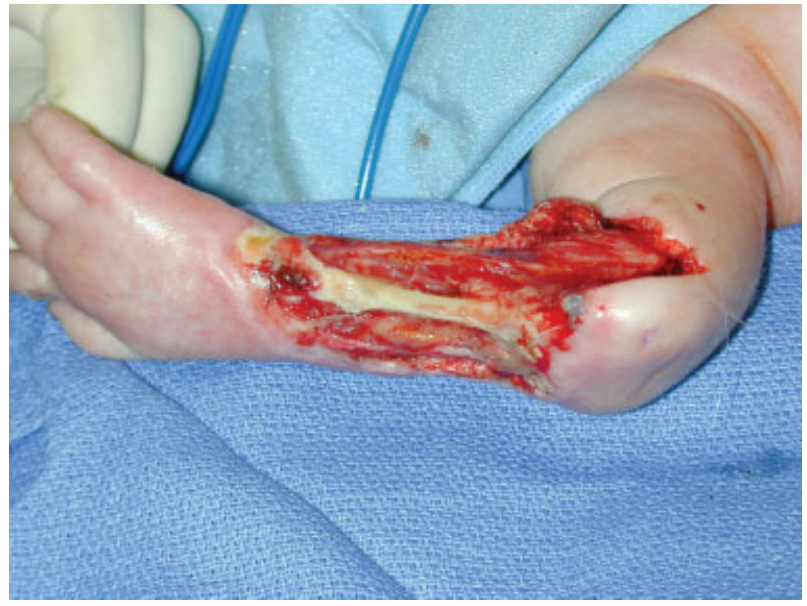

Fig. 1 Patient's right forearm after definitive debridement with exposed ulna, ulnar nerve, and artery.

note, the patient was insensate from birth in his ulnar and median nerve distributions. After 2 weeks and several more debridements, the patient was taken to the OR for definitive treatment. Removal of all nonviable tissue resulted in a defect of $60 \%$ of his forearm skin and musculature with exposed ulna, ulnar nerve, and artery ( - Fig. 1). Planned treatment was a two-stage operation utilizing a rectus abdominis muscle as a pedicled flap for soft tissue coverage and dividing the flap 2 to 3 weeks after placement. However, intraoperatively after dissection of the rectus abdominis muscle, it was noted that the muscle as a pedicled flap was too short to cover the soft tissue defect. Therefore, the planned delayed flap was converted to a free flap. During the operation, the patient's ulnar and median nerves were completely neurolysed using an operating microscope, and the rectus abdominis muscle flap was isolated. The muscle was $10 \mathrm{~cm}$ long with a pedicle length of $8 \mathrm{~mm}$ and arterial pedicle diameter of $0.8 \mathrm{~mm}$ (-Fig. 2). The flap artery was sewn in end-to-side to the proximal ulnar artery and the two veins were anastomosed end-to-end to the ulnar comitantes using 10-0 nylon suture

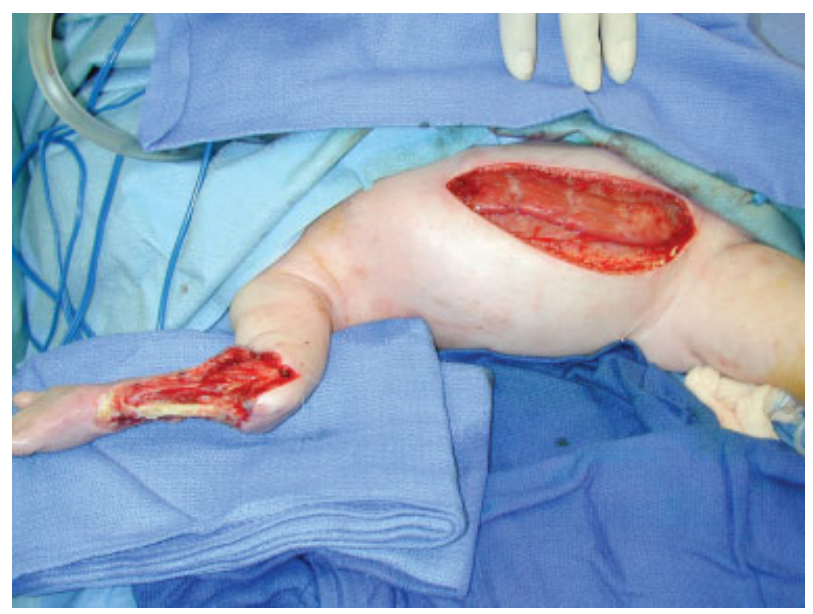

Fig. 2 Dissection of the rectus abdominis muscle. The muscle was $10 \mathrm{~cm}$ long with a pedicle length of $8 \mathrm{~mm}$ and the pedicle diameter was $0.8 \mathrm{~mm}$.

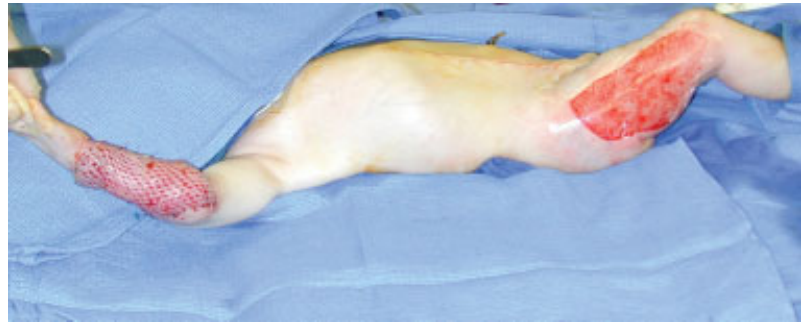

Fig. 3 Skin graft harvest from the right thigh.

with a tapered edge atraumatic needle. Interrupted sutures were used in arterial and venous anastomoses. The patient remained intubated in the neonatal intensive care unit to remain immobilized. Postoperatively, aspirin was given as the only anticoagulant. The patient was taken back to the operating room 7 days later for skin grafting of the muscle. The graft was harvested from the buttock after saline clysis of the region to expand the area (-Fig. 3). The flap and graft survived without complication and the patient went on to demonstrate a completely healed wound with a return in median and ulnar nerve sensation. He continued to undergo intermittent operations to release ulnar-sided contractures. His long-term follow-up shows good flap and skin graft take (-Fig. 4).

\section{Discussion}

Microsurgery has been slower to develop in pediatric population compared with the adult population due to technical difficulties inherent in the small size of the vessels, prolonged anesthesia, and difficult postoperative care. However, given advancements in microsurgical and anesthetic techniques, these problems can be overcome. With advances in supermicrosurgery, smaller caliber vessels ranging from 0.3 to $0.8 \mathrm{~mm}$ can be anastomosed., ${ }^{3,4}$ The question of whether maternal circulating procoagulants are present at birth has also been raised. These might predispose a flap to thrombosis.

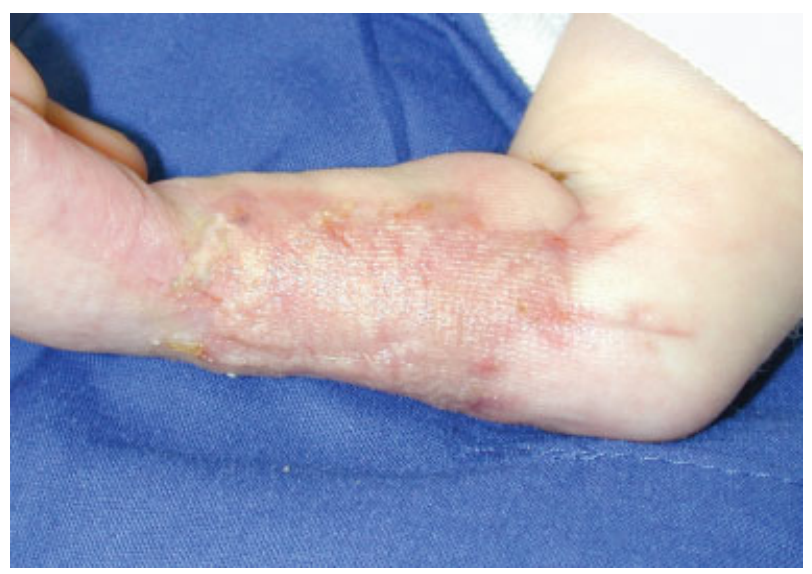

Fig. 4 Long-term follow-up showing good flap and graft take. 
In neonatal patients, the absence of associated comorbid factors and unscarred clear anatomy are encouraging. However, although technical challenges can be overcome, finding adequate donor sites can be challenging. In our patient, there were few suitable donor sites due to the need for a long and wide muscle with a reliable vascular pedicle of sufficient size: latissimus dorsi or rectus abdominis. Given the age of the patient, vascular caliber and thrombosis was a concern; therefore, initially the free flap was not considered as a treatment option. A delayed flap was the preferred treatment and therefore the rectus abdominis was chosen. Because the pedicled rectus abdominis flap failed to adequately cover the forearm defect, it was converted to a free flap. There was no vasospasm or thrombotic complications noted postoperatively.

\section{Conclusion}

Aggressive treatment of large soft tissue losses can be undertaken with the knowledge that free tissue transfer in the neonate is feasible despite small vascular caliber.
An experienced surgical team remains one of the most important factors to obtain a good outcome, and knowledge of multiple reconstructive options in neonates appears essential for satisfactory results.

Funding

None.

\section{References}

1 Ohmori K, Harii K, Sekiguchi J, Torii S. The youngest free groin flap yet? Br J Plast Surg 1977;30(4):273-276

2 Parry SW, Toth BA, Elliott LF. Microvascular free-tissue transfer in children. Plast Reconstr Surg 1988;81(6):838-840

3 Koshima I, Nanba Y, Tsutsui T, Takahashi Y. Medial plantar perforator flaps with supermicrosurgery. Clin Plast Surg 2003; 30(3):447-455, vii

4 Koshima I, YamamotoT, Narushima M, Mihara M, Iida T. Perforator flaps and supermicrosurgery. Clin Plast Surg 2010;37(4):683-689, vii-iii 